\title{
Diagnóstico de fenilquetonuria en Chile
}

\author{
Dra. Marta Colombo C. 1 ; Dra. Ledia Troncoso A. ${ }^{1}$; Dra. Ena Raimann B. ${ }^{1}$; \\ Psicol. Carmen Gloria Perates ${ }^{2}$; T. L. Teresa Barros H. ${ }^{3}$; Nutr. Verónica Cornejo E. ${ }^{4}$
}

Phenylketonuria: Late diagnosis

The cinical and biochemjcal characteristics of 44 PKU children, diagnosed at an average age of 3 years 11 months is presented. $90 \%$ of these children consulted because of delayed psychomotor development or mental retardation: $100 \%$ of them showed hypopigmented skin, but only $32 \%$ had bright colored eyes. $86 \%$ had a peculiat must odor. The neurological signs and symptoms were predominantly hyperactivity, irritability, hypotonia and hyperreflexia. Ferric choride and 2-4 dinitrophenylhydrazine tests were positive in all patients. Paper chromatography of amino acids demonstrated levels of phenylalanine over $20 \mathrm{mg} \%$. The importance of making an early diagnosis and treatment in order to prevent mental retardation is emphasized. The need to develop newborn screening for PKU in our country is raised.

(Key words: phenylketonuria, PKU.)

La fenilquetonuria (PKU) es un error congénito del metabolismo del aminoácido esencial fenilalanina (FA), cuyo exceso afecta al sistema nervioso en desarrollo, to que se manifiesta fundamentalmente por retardo mental (RM). Su herencia es autosómica recesiva y su incidencia de $1: 10.000$ recién nacidos vivos ${ }^{1}$. Fölling la describió por primera veź en 1934, al encontrar aumento de ácido fentlpirúvico en la orina de 2 hermanos con RM profundo y un olor característico. El denominó a esta enfermedad "Idiocia fenilpirúvica"2. Tres años más tarde, Penrose y Quastel sugiririeron el nombre de "fenilquetonuria", que se mantiene en la actualidad ${ }^{3}$. Bickel, en 1953, pudo demostrar que al restringir la FA en la dieta a los pacientes con $\mathrm{PKU}$, éstos presentaban mejoría bioquímica y clinica ${ }^{4}$. Posteriormente se pudo comprobar estrecha relación entre la edad de comienzo del tratamiento nutricional $y$ el desarrollo intelectual de estos pacientes, determinándose que en aquellos nirnos que se sometían a dieta especial en los primeros meses de vida, su desarrollo intelectual era normal ${ }^{5}$.

Basado en estos hechos, Guthrie en 1961 planteó la necesidad de hacer un diagnóstico

1. Médico Unidad Neuropsicología, INTA.

2. Psicóloga, Unidad Neuropsioología, INTA.

3. Técnico de Laboratorio, Unidad de Neuropsicología, INTA.

4. Nutricionista, Linidad de Neuropsicología, INT A. masivo y precoz de esta enfermedad, para lo cual desarrolló un método simple para medir fenilalaninemia ${ }^{6}$ y establecer programas de búsqueda masiva o rastreo, que se aplican a todo RN. En paises industrializados, donde el rastreo neonatal se emplea desde hace más de 20 años, la PKU es considerada una importante causa prevenible de $\mathrm{RM}^{\text {? }}$.

La hiperfenilalaninemia (HFA), característica de esta enfermedad, se produce por deficiencia total o parcial de la enzima hepática fenilalanina hidroxilasa o de su sistema cofactor (dehidropteridina reductasa), que producen falla en la conversión de FA a tirosina, induciendo aumento de la FA. El exceso de este aminoácido abre vías metabólicas normalmente inactivas, produciéndose catabolitos neurotóxicos, entre éstos el ácido fenilpirúvico ${ }^{1}$ (figura 1 ).

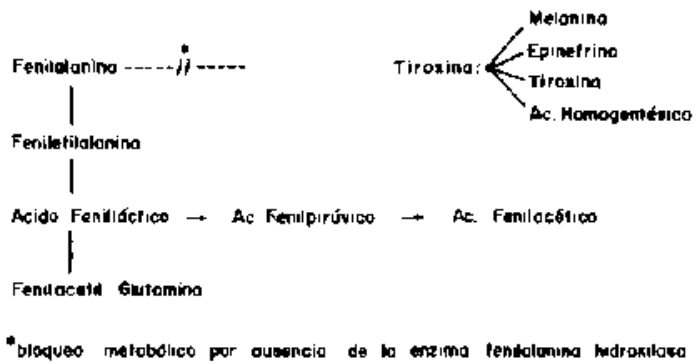

Figura 1. Vía metabólica de la ferilalanina (FA) en la fenilquetonuria (PKU). 
Tabla 1

C3asificación de hiperfenilalaninemia (HF A)

\begin{tabular}{|c|c|c|c|c|}
\hline Carácter & Déficit enzimático & FA sangujinea & Características clínicas & Tratamiento \\
\hline $\begin{array}{l}\text { PKU } \\
\text { clásica }\end{array}$ & $\begin{array}{l}\text { Ausencia fenilalanina } \\
\text { hidroxilasa }\end{array}$ & $>20 \mathrm{mg} \%$ & $\begin{array}{l}\text { RM y estigmas } \\
\text { caracter ísticas }\end{array}$ & $\begin{array}{l}\text { FA } 250-500 \\
\mathrm{mg} / \mathrm{día}\end{array}$ \\
\hline $\begin{array}{l}\mathrm{HFA} \\
\text { persistente }\end{array}$ & $\begin{array}{l}>3 \% \text { actividad de } \\
\text { fenilalanina hidroxilasa }\end{array}$ & $\begin{array}{l}\text { Inicio } 20 \mathrm{mg} \% \\
\text { luego }<10 \mathrm{mg} \%_{0}\end{array}$ & Normal & Ninguno \\
\hline $\begin{array}{l}\text { HFA } \\
\text { transitoria }\end{array}$ & $\begin{array}{l}\text { Madurez enzimática } \\
\text { retardada }\end{array}$ & $\begin{array}{l}\text { Inicio }<16 \mathrm{mg} \% \\
\text { cae bruscamente }\end{array}$ & Normal & $\begin{array}{l}\text { Dieta hipoproteica. } \\
\text { Suplementación } \\
\text { Vit. C }\end{array}$ \\
\hline $\begin{array}{l}\text { Déficit de } \\
\text { dehidropteridina } \\
\text { reductasa }\end{array}$ & $\begin{array}{l}\text { Déficit cofactor } \\
\text { dehiđaropteridina } \\
\text { Ieductasa }\end{array}$ & $>20 \mathrm{mg} \%$ & $\begin{array}{l}\text { RM y estigmas } \\
\text { características }\end{array}$ & $\begin{array}{l}\text { No responde dieta } \\
\downarrow \text { FA } \\
\text { Administración } \\
\text { cofactor }\end{array}$ \\
\hline$-\underset{\text { Materna }}{\text { PKU }}$ & $\begin{array}{l}\text { PKU clásica en la } \\
\text { madre }\end{array}$ & $>10 \mathrm{mg} \%$ & $\begin{array}{l}\text { Microcefalia, RM. } \\
\text { Cardiopatía congénita } \\
\text { en el feto }\end{array}$ & $\begin{array}{l}\text { Dieta igual. } \\
\text { PKU clásica }\end{array}$ \\
\hline
\end{tabular}

Actualmente se describen 5 variedades clínicas de HFA (tabla I). Dentro de éstas, la PKU clásica es la más estudiada. Clínicamente la enfermedad se catacteriza por hipopigmentación de piel, pelo e iris, siendo clásica la descripción de estos niños como muy blancos, rubios y de ojos claros. Los síntomas pueden comenzar en los primeros meses de vida, especialmente con vómitos, eccema rebelde a tratamiento, falta de interés por el medio, convulsiones, frecuentemente espasmos masivos y un olor muy particular a humedad. Alrededor de los 6 meses de edad se hace eviden. te la presencia de retraso del desarrollo psicomotor. En el niño mayor aparecen graves trastornos de conducta como agresividad, rabietas, hiperactividad. Pueden también presentar autismo. En esta forma de PKU los niveles plasmáticos de FA están sobre los $4 \mathrm{mg} \%$ (normal: 2 a $4 \mathrm{mg} \%$ ) desde las 48 horas de vida del RN, lo que permite su pesquisa precoz ${ }^{8,10}$.

\section{MATERIAL. Y METODO}

La muestra está compuesta por $\mathbf{4 4}$ niños PKU enviados a la unidad de neuropsicología, INTA, Universidad de Chile, entre los años 1973 y 1985 para estudio metabólico. En estos pacjentes se planteó la sospecha de PKU utilizando en orina las pruebas cualitativas de cloruro férrico (la orina adquiere color verde) y $2-4 \mathrm{di}$ nitrofenilhidrazina (en la orina se forma un precipitado amarillo), detectando la presencia de ácido fenilpi rúvico y quetoácidos respectivamente ${ }^{\mathbf{1}}$.
La confîrmación del diagnóstico se realizó mediante determinación sernicuantitativa de FA por cromatografía en papel de sangre y orina, en algunos casos cuantitativa, usando un analizador de aminoácidos.

La evaluación psicométrica de estos niños se hizo con las pruebas de Binet-Kuhlman y Gesell 12, 35. Una vez establecido el diagnóstico, se inició tratamiento nut ricional, conșistente en una dieta con bajo contenido de FA (19.5 a $35 \mathrm{mg} F \mathrm{~A} / \mathrm{kg} x$ día) y un aporte adecuado de proteínas y calorías (tabla 2). Esta dieta permite mantener niveles plasmáticos de FA entre $3 y$ $8 \mathrm{mg} \mathrm{m}^{13}$.

Tabla 2

Requerimientos de FA, proteínas y calorías para niños $P K U$

\begin{tabular}{cccc}
\hline $\begin{array}{l}\text { Edad } \\
\text { (meses) }\end{array}$ & $\begin{array}{c}\text { FA } \\
\text { (mg/kg dia) }\end{array}$ & $\begin{array}{c}\text { Proteinas } \\
\text { (g/kg dia) }\end{array}$ & $\begin{array}{c}\text { Calorías } \\
\text { (kcal/kg dia) }\end{array}$ \\
\hline $0-3$ & $70-90$ & 2,5 & 120 \\
$4-6$ & $60-70$ & 2,5 & 115 \\
$7-9$ & $40-50$ & 2,5 & 110 \\
$10-12$ & $30-40$ & 2,5 & 105 \\
& & & \\
Años & & Total g/dia & Kcal/dia \\
& & & \\
$1-2$ & 25 & 25 & 1.300 \\
$3-4$ & 24 & 25 & 1.300 \\
$5-6$ & 20 & 30 & 1.300 \\
$7-8$ & 18 & 30 & 1.800 \\
$9-10$ & 17 & 35 & 2.000 \\
$11 \mathrm{y}+$ & 15 & 40 & 2.000 \\
\hline
\end{tabular}

Fuente: John Hopkins Hospital. Nutritional Management (13). 


\section{RESULTADOS}

Los resultados se refieren sólo a los hallazgos en el momento de hacer el diagnóstico. El promedio de edad al confirmar la PKU fue de 3 años 11 meses (rango 11 meses a 12 antos). Cabe destacar, sin embargo, que las madres notaron retraso del desarrollo psicomotor (RDPM) de su hijo ya a los 8 meses de edad, to que se hizo francamente evidente para ellas a los 14 meses de vida.

En $13,6 \%$ de estos pacientes habia antecedentes de consanguinidad, en igual porcentaje exist ia algún familiar portador de PKU, en 4,5\% antecedentes de trastornos del aprendizaje y, en una proporción del $2,2 \%$ para cada hecho, retardo mental, síndrome de Down, sindrome de Turner y sordomudez en algún pariente.

El motivo de consulta fue, en $90 \%$ de estos nifios, retardo del desarrollo psicomotor aislado $(77,6 \%)$, asociado a convulsiones $(9 \%)$, retardo mental $(4,5 \%)$, convulsiones $(2,2 \%)$ y retardo del lenguaje $(4,5 \%)$. No se obtuvo información en $4,5 \%$ de los casos.

En el examen físico todos los nifios presentaban una piel muy blanca, $64 \%$ cabellos claros, $32 \%$ ojos claros, $34 \%$ eccentas. El olor a humedad estaba presente en $86 \%$ de los casos. En el examen neurológico, $68 \%$ de ellos presentaba hiperactividad; $68 \%$ initabilidad; $43,1 \%$ hipotonía con hiperreflexia y $94,2 \%$ retraso psicomotor o mental.

La evaluación psicométrica demostró retraso del desarrollo psicomotor leve y moderado en $11,8 \%$ de los niños y RM en $82,4 \%$, quedando en la categoría de limítrofe sólo $5,8 \%$ de los niños (tabla 3).

Las reacciones de cloruro férrico y 2-4 dinitrofenilhidrazina fueron positivas en todos los casos y la cromatografia en papel de aminoácidos demostró concentraciones elevadas de FA $>20 \mathrm{mg} \%$ ) en todos los pacientes.

Tabla 3

Fvaluación psicométriç en 44 niños PKU (Escala Binet-Kuhlman y Gesell)

\begin{tabular}{lr}
\hline & \multicolumn{1}{c}{$\%$} \\
\hline RDPM leve & 5,9 \\
RDPM moderado & 5,9 \\
RM leve & 8,8 \\
RM cducable & 26,5 \\
RM entrenable & 14,7 \\
RM custodiable & 32,4 \\
Limítrofe & 5,8 \\
\hline
\end{tabular}

\section{DISCUSION}

E1 RM es considerado como un problema de salud pública, por las graves secuelas que provoca en el individuo, la familia y la sociedad ${ }^{17}$. En 1962 la Organización Mundial de la Salud (OMS) determinó que un 3\% de la población mundial padecía algún grado de RM. Sin embargo, cabe señalar que múltiples investigaciones realizadas en los últimos años han determinado que $50 \%$ de las causas que inducen a este daño pueden prevenirse: entre ellas han adquirido relevancia los errores congénitos del metabolismo.

Es asi como en los países en que se aplican programas masivos de detección precoz de PKU en el periodo neonatal, ésta ha desaparecido como causa de RM. En Chile, en que aún no se establecen programas de este tipo, $y$ basándonos en que la incidencia sería, como en otras partes del mundo, de 1:10.000 recién nacidos vivos, cada an̂o nacetian 28 niños PKU, que de no ser diagnosticados y tratados, sufririan RM.

La falta de signos clínicos específicos de la $\mathrm{PKU}$ en los primeros meses de vida hace difícil su diagnóstico precoz. Sin embargo, se debe enfatizar que frente a hallazgos como vómitos persistentes, eccemas rebeldes a tratamiento, presencia de espasmos masivos, olor especial a humedad, RDPM junto con hipopigmentación de piel, pelo e iris, debe hacer sospechar la enfermedad y solicitar al menos una reacción de cloruro férrico y $2-4$ dinitrofenilhidrazina en orina.

Como se demostró en esta muestra, las madres notaron RDPM de sus hijos a los 8 meses de edad, haciéndose el diagnóstico de PKU, en promedio, a los 3 anfos 11 meses. En este sentido, cabe recalcar que existe una estrecha relación entre edad de iniciación del tratamiento nutricional y desarrollo de capacidad intelectual.

Se ha demostrado que se logra una inteligencia dentro de limites normales sólo en aquellos niños que comienzan un tratamiento antes de los 3 meses de vida ${ }^{2}, 4,27,28$. Idealmente éste debe hacerse desde el primer mes de edad, ya que los niveles elevados de FA y sus metabolitos son neurotóxicos para el sistema nervioso en desarro$110^{19}, 20$. En un estudio colaborativo realizado en 111 niños PKU diagnosticados entre los 3 y 92 días de edad y evaluados 4 años después, se pudo demostrar que su cuociente intelectual (CD) promedio era de 93 (escala Binet). Sin embargo, al separarlos por edad de inicio del tratamiento, en 
aquellos nifios en que éste comenzaba antes de los 30 dias de edad el CI promedio era de 95; en cambio, sólo alcanzaba 85 puntos cuando el tratamiento se instauraba entre los 31 y 35 d $\operatorname{jas}^{29}$. Estos antecedentes determinan la urgencia de hacer un diagnóstico y tratamiento pre. coces.

Dentro de los métodos de diagnóstico de PKU las reacciones de cloruro férrico y 2.4 dinitrofenilhidrazina son de muy bajo costo y fácil ejecución; sin embargo, generalmente se alteran sólo después del primer mes de vida de] niño ${ }^{24}$. Por esta razón el diagnóstico en el período neonatal se basa en la determinación de FA, ya sea por el método de Guthrie (inhibición bacteriana) en sangre, o por cromatografía en papel o capa fina en orina ${ }^{21,23}$.

Es así como se ha creado la necesidad de ejecutar el diagnóstico masivo de PKU a todos los $\mathrm{RN}$, con el fin de instaurar el tratamiento nutricional en forma precoz.

La terapia nutricional requiere de un control estricto, el que se realiza a través de controles bioquímicos seriados, a modo de mantener los niveles séricos de FA entre 3 y 8 ing\%. Esto implica suspender todas las proteínas de alto valor biológico, requiriéndose sustitutos lácteos pobres en FA para garantizar un optimo desarrollo pondoestatural ${ }^{13,15}$.

El diagnóstico de PKU en nuestro país es tardío y ello no ha variado con los afios, por lo que parece importante insistir, por un lado, en solicitar los exámenes para descartar PKU en todo niño con las características físicas descritas, retraso del desarrollo, espasmos masivos y olor especial, lo más precozmente posible $\mathrm{y}$, por otro lado, apoyar el desarrollo de un sistema de rastreo masivo de PKU en el RN, de modo de prevenir el RM por esta causa.

\section{RESUMEN}

Se presentan las características clínicas y bio. químicas de 44 niños PKU diagnosticados tardiamente, a una edad promedio de 3 años 11 meses. Se enfatiza la importancia de hacer un diagnóstico y tratamiento oportuno, de modo de prevenir la aparición de retardo mental. Se plantea la necesidad de desarrollar un programa de búsqueda masiva para la detección de PKU en el período neonatal en nuestro país.

\section{REFERENCLAS}

1. Sicnbury J., Wyngarden J., Fredrickson D.: Part 3. Diseases related primarily to disorders of amino acid metabolism. In: The Metabolic Basis of Inherited Disease. Stanbury J., Wyngaarden J., Fredrickson D. (eds). 4th. ed. "A Blakiston Publication", USA, $1981 ; 206-256$.

2. Scriver CA. Rosemberg L.: En: Metabolismo de los aminoácidos y sus trastornos. Editor: Sabater J. Editorial Científico-Médica. Barcelona, España, 1979

3. Bitkel H., Gerrard A.J., Hickman E.M.: Influence of phenyl alanine intake on phenylketonuria. Lancet 1953; 2: 812-813.

4. Rosemberg I.E., Scriver C.R.: Disorders of amino acids metabolism. In: Duncan's Diseases of Metabolism. Bondy P.K. and Rosemberg G.W. (ads) W.B. Sounders. Philadelphia. London and Toronto $1974 ; 497.610$.

5. Scriver Ch., Clow C': Medical progress phenylketonuria epitome of human biochemical genetics (First of Two Parts). New Engl J Med 1980;303 (23): 1336-1342.

6. Guthrie $R$, Susi A.: A simple phenylalanine method for detecting phenylketonuria in large populations of newborn infants. Pediatrics 1963; 32: 338-343.

7. Guthrie $R$ : Mass Screening for Genetic Disease, Hosp Pract 1972; 93: 100.

8. Mamunes P.: Neonatal screening test. Ped Clin North Am $1980 ; 27(4)$ : 733-751.

9. Holtzman $N$.: Newborn screening for inborn of metabolism. Ped Clin North Am 1978; 25(3); $411-420$.

10. Guthrie $R$.: Phenylketonuria screening pfograms. New Engl J Med 1963; 269; 52-58.

11. Howell R., Holtzman N., Thomas G.: One dimension chromatographic procedure for amino acids in serum. In: Selected screening test for genetic metabolic diseases. The John F. Kennedy Instimte for Habilitation of the Mentally and Physically Handicapped Child (ed), Baltimore, Maryland, 1976: $1-8$.

12. Kuhlman-Binet: Escala de Inteligencia BinetSimon. Revisión Kuhlman-Binet, 1912.

13. Valle D., Cavarnaro B.: Phenylketonuria: In: Dietary management of inborn errors of metabolism. The John Hopkins Hospital Nutritional Management (eds). Maryland 1984; 295-314

14. Coutis J.: Simposium of dietary management of disease: The dictary management of phenylketonuria. Proc Nutr Soc 1979; 38: 315-320.

15. Amstrong M.D., Tyler F.M.: Studies on phenylketonuria. I. Restricted phenylalanine intake in phenylketonuria. Nutr Rev 1983; 41 (1): 15 .

16. Flannery D.B. et al.: Dietary management of phenylketonuria from birth using a phenylalaninefree product. J Pediatr 1983; $103(2) \cdot 247-249$.

17. Carter Ch.: Capítulo XVI. Procesos que responden al tratamiento o a la prevención. En: Retraso mental en el nirio. Editorial Pediátrica, Barcelona, España, 1973. 
18. Fliman M.: Un diagnóstico de la situación de la salud mental en Chile. Rev Med Chile 1972; 100: 343-347.

19. Republica de Chile. Ministerio de Economia. Fo mento y Reconstrucción. Instituto Nacional de Estad isticas. Demografía. 1982. Santiago, Chile.

20. Menkes J.H.: The patinogenesis of mental retardation in phenylketonuria and other inborn errors of amino acid metabolism. Pediatrics 1967: 39: 29?302.

21. Guttler $F_{1}$ : Phenylketonuria: 50 years since Folling discovery and still expanding our clinical and biochemical knowledge. Acta Paediatr Scand 1964; 73(6): 705-16. (50 ref.).

22. Meryash D., Levy $H$. , Guthrie $R$., Warner $R$., Bloom S. Care J.: Prospective study of early neonatal sureening for phenylketonuria. Frechette A., Russo P. (eds). New Engl J Med 1981; 304: 294. 296.

23. Starfield B., Holtzman N.: A comparison of effectjveness of screening for phenylketonuria in the United States. United Kingdom and Ireland. New Engl I Med 1975; 293(3): 118-121.

24. Holziman N., Meck A., Mellits D.: Neonatal screening for phenylketonuria. I. Effectiveness. JAMA 1974; 229: 667-670.

25. Bradley D.M.: Screening for inhetited metabolic disease in Wales using urine impregnated filter paper. Arch Dis Child 1975; \$0(4): 264.268. (Abstract. 2451).

26. Guthrte R., Womer R., Spon R., Raltaziur R.: Laying the myth of the "Slow rise" of phenyl- alanine blood levels in classical phenylketonuria. Pediat Res 1980; 14 (Part 2): 522-524.

27. Edword R.P., Cabe Mc., (rayl R.: Newborn screening for phenylketonuria (PKU). When should the infant be tested? Pediat Res 1981; 15(4): 565 . (Part 2).

28. Williamson M., Koch R. Azen C., Chary C.: Correlates of intelligence test results in treated phenylketonuric children. Pediatrics 1981; 68(2): 161167.

29. Dobsan J., Koch R., Whlamson M. et al, Cognitive development and dietary therapy in phenylketonuric children. New Engl J Med 1968; 278: 1142-1144.

30. Dobson J., Williamson $M$. . Azen C., Koch R.: Intellectual assesment of 111 four year old children with phenylketonuria. Pediatrics $1977 ; 60(6)$ : 822827

31. Kang E.S., Sollec N.. Gerald P.: Results of treatment and termination of the diet plenylketonuria (PKU). Pediatrics 1970; 46(6): 881-890.

32. Seashore M.R. et al.: Loss of intellectual function in children with phenylketonuria after relaxation of dietary phenylalanine restriction. Pediatrics $1985 ; 75(2): 226-232$.

33. Koch $R$. et al:: Preliminary report on the effects of diet discontinuation in PKU. J Pediatr 1982;100 (6): 870-875. (Abstract 651).

34. Koff $E$., Kammerer B., Boyle P., Pireschel $S$. Intelligence and phenylketonuria: Effects of diet termination. J Pedjatr 1979; $94(4)$ : 534-537.

35. Gesell A., Amotnda $C$.: Developmental Diagnosis. New York. Hoeber $1941 ; 18$. 